\title{
How Calogero-Moser particles can stick together
}

\author{
A. Zabrodin*
}

January 2021

\begin{abstract}
We show that the configuration in the phase space of the elliptic Calogero-Moser model when particles stick together in pairs is stable under the third Hamiltonian flow of the model. The equations of motion for the pairs coincide with the equations of motion for poles of elliptic solutions to the B-version of the KadomtsevPetviashvili equation.
\end{abstract}

\section{Introduction}

This paper is a short remark on how the equations of motion for poles of elliptic solutions to the B-version of the Kadomtsev-Petviashvili (BKP) equation can be obtained entirely in terms of the elliptic Calogero-Moser model [1, 2, 3, 4]. Since Krichever's seminal paper [5] it is known that poles of elliptic solutions to the KP equation move as particles of the elliptic Calogero-Moser many-body system. At the same time, solutions to the BKP equation [6, 7] are those solutions to the KP equation,

$$
3 u_{y y}=\left(4 u_{t}-12 u u_{x}-u_{x x x}\right)_{x},
$$

for which the tau-function, i.e the entire function $\tau=\tau(x, y, t)$ such that

$$
u=\partial_{x}^{2} \log \tau
$$

at $y=t_{2}=0$ is a full square, i.e., all its zeros are of the second order. Poles of the elliptic solutions are zeros of the tau-function which, therefore, can be identified with coordinates of the Calogero-Moser particles. This means that equations of motion for poles of solutions to the BKP equation, which are elliptic functions in $t_{1}=x$, derived in [8] should follow from restriction of the third Hamiltonian flow $t=t_{3}$ of the CalogeroMoser model to the subspace of the phase space in which $N=2 n$ particles stick together in pairs, i.e. their coordinates satisfy $x_{2 i-1}=x_{2 i}, i=1, \ldots, n$. (However, one can not directly put $x_{2 i-1}=x_{2 i}$ in the equations of motion because this limit is singular; instead, one should put $x_{2 i}-x_{2 i-1}=O(\varepsilon)$ and consider the limit $\varepsilon \rightarrow 0$.) As is easily seen, this configuration is immediately destroyed under the standard (second) Hamiltonian

*Steklov Mathematical Institute of Russian Academy of Sciences, Gubkina str. 8, Moscow, 119991, Russian Federation, e-mail: zabrodin@itep.ru 
flow $y=t_{2}$ of the Calogero-Moser model. Nevertheless, we show that it is stable under the third Hamiltonian flow, derive equations of motion for the pairs and show that they coincide with the equations of motion for poles of elliptic solutions to the BKP equation obtained in [8].

The elliptic $N$-body Calogero-Moser system is known to be integrable: it has $N$ integrals of motion (Hamiltonians) in involution. The first three are (see, e.g., [9])

$$
\begin{aligned}
& H_{1}=-\sum_{i=1}^{N} p_{i}, \\
& H_{2}=\sum_{i=1}^{N} p_{i}^{2}-\sum_{i \neq j} \wp\left(x_{i}-x_{j}\right), \\
& H_{3}=-\sum_{i=1}^{N} p_{i}^{3}+3 \sum_{i \neq j} p_{i} \wp\left(x_{i}-x_{j}\right),
\end{aligned}
$$

where $\wp(x)$ is the elliptic Weierstrass $\wp$-function with complex periods $2 \omega_{1}, 2 \omega_{2}$ such that $\operatorname{Im}\left(\omega_{2} / \omega_{1}\right)>0$. The standard Hamiltonian of the Calogero-Moser system is $H_{2}$. As is claimed above (see [5, 10]), the Hamiltonian equations

$$
\partial_{t_{a}} x_{i}=\frac{\partial H_{a}}{\partial p_{i}}, \quad \partial_{t_{a}} p_{i}=-\frac{\partial H_{a}}{\partial x_{i}}, \quad a=2,3
$$

are equations of motion for poles $x_{i}$ of elliptic solutions

$$
u(x, y, t)=-\sum_{i=1}^{N} \wp\left(x-x_{i}(y, t)\right)+\mathrm{const}
$$

to the KP equation (11) as functions of $t_{2}=y$ and $t_{3}=t$. For the BKP equation, the $t_{2}$-flow is frozen and we will restrict the $t_{3}$-dynamics to the subspace of the phase space with $x_{2 i-1}=x_{2 i}, i=1, \ldots, n$.

\section{Equations of motion for pairs of Calogero-Moser particles}

Let the number of Calogero-Moser particles $N$ be even, $N=2 n$. We assume that the particles are joined in $n$ pairs, so that the coordinates of two particles in each pair tend to each other:

$$
x_{2 i}-x_{2 i-1}=\varepsilon \delta_{i}, \quad \varepsilon \rightarrow 0, \quad i=1, \ldots, n .
$$

Here $\delta_{i}=O\left(\varepsilon^{0}\right)$ as $\varepsilon \rightarrow 0$. We define a submanifold $\mathcal{B}_{n}(\varepsilon)=\mathcal{B}_{n}\left(\varepsilon,\left\{\delta_{i}\right\}\right)$ in the $2 N=$ $4 n$ dimensional phase space of the Calogero-Moser model by imposing the following conditions on the momenta:

$$
\begin{aligned}
& p_{2 i-1}=\frac{1}{\varepsilon \delta_{i}}+\alpha_{i} \varepsilon+\beta_{i} \varepsilon^{2}, \\
& p_{2 i}=-\frac{1}{\varepsilon \delta_{i}}-\alpha_{i} \varepsilon+\beta_{i} \varepsilon^{2}
\end{aligned}
$$


(together with the conditions ([6]) ), where

$$
\alpha_{i}=\alpha_{i}(\varepsilon)=\alpha_{i, 0}+\alpha_{i, 1} \varepsilon+\alpha_{i, 2} \varepsilon^{2}+\ldots, \quad \beta_{i}=\beta_{i}(\varepsilon)=\beta_{i, 0}+\beta_{i, 1} \varepsilon+\beta_{i, 2} \varepsilon^{2}+\ldots
$$

are some series in $\varepsilon$. Given $\delta_{i}$, the $\beta_{i}$ will turn out to be fixed (as we shall see) and so the submanifold $\mathcal{B}_{n}(\varepsilon)$ is $2 n$-dimensional with coordinates $x_{2 i-1}, \alpha_{i}, i=1, \ldots, n$.

We are going to show that the space $\mathcal{B}_{n}(\varepsilon)$ is preserved by the $t_{1^{-}}$and $t_{3}$-flows as $\varepsilon \rightarrow 0$ (conjecturally, it is preserved by all "odd" flows). For the $t_{1}$-flow $\partial_{t_{1}} x_{i}=-1$, $\partial_{t_{1}} p_{i}=0$ this is obvious. The $t_{3}$-flow is given by

$$
\left\{\begin{array}{l}
\dot{x}_{i}=-3 p_{i}^{2}+3 \sum_{j \neq i} \wp\left(x_{i}-x_{j}\right), \\
\dot{p}_{i}=-3 \sum_{j \neq i}\left(p_{i}+p_{j}\right) \wp^{\prime}\left(x_{i}-x_{j}\right),
\end{array}\right.
$$

where dot means the $t_{3}$-derivative. We have by inspection:

$$
\dot{x}_{2 i-1}=-6 \frac{\alpha_{i}}{\delta_{i}}+6 \sum_{j \neq i} \wp\left(x_{2 i-1}-x_{2 j-1}\right)+O(\varepsilon)
$$

and for consistency we should require that

$$
\dot{x}_{2 i}-\dot{x}_{2 i-1}=\varepsilon \dot{\delta}_{i}
$$

i.e., that evolution in $t_{3}$ does not generate non-vanishing terms as $\varepsilon \rightarrow 0$ (this means that the particles remain to be stuck together). We have from the first equation in (8)):

$$
\begin{aligned}
\partial_{t_{3}}\left(x_{2 i}-x_{2 i-1}\right)= & 3\left(p_{2 i-1}^{2}-p_{2 i}^{2}\right) \\
& +3 \sum_{j \neq i}\left(\wp\left(x_{2 i}-x_{2 j-1}\right)+\wp\left(x_{2 i}-x_{2 j}\right)\right) \\
& -3 \sum_{j \neq i}\left(\wp\left(x_{2 i-1}-x_{2 j-1}\right)+\wp\left(x_{2 i-1}-x_{2 j}\right)\right) .
\end{aligned}
$$

Taking into account that $p_{2 i-1}^{2}-p_{2 i}^{2}=4 \beta_{i} \delta_{i}^{-1} \varepsilon+4 \alpha_{i} \beta_{i} \varepsilon^{3}$, we obtain, expanding the right hand side in powers of $\varepsilon$, that (10) is true indeed and

$$
\begin{gathered}
\dot{\delta}_{i}=12 \beta_{i, 0} \delta_{i}^{-1}+6 \delta_{i} \sum_{j \neq i} \wp^{\prime}\left(x_{2 i-1}-x_{2 j-1}\right) \quad \text { in order } \varepsilon \\
4 \beta_{i, 1}=\delta_{i}^{2} \sum_{j \neq i}\left(\delta_{j}-\delta_{i}\right) \wp^{\prime \prime}\left(x_{2 i-1}-x_{2 j-1}\right) \quad \text { in order } \varepsilon^{2} \\
24 \alpha_{i} \beta_{i, 0} \delta_{i}+24 \beta_{i, 2}+\delta_{i}^{2} \sum_{j \neq i}\left(2 \delta_{i}^{2}+3 \delta_{j}^{2}-3 \delta_{i} \delta_{j}\right) \wp^{\prime \prime \prime}\left(x_{2 i-1}-x_{2 j-1}\right)=0 \quad \text { in order } \varepsilon^{3} .
\end{gathered}
$$

Next, we write the second equation in (8):

$$
\begin{gathered}
\dot{p}_{2 i-1}=-3\left(p_{2 i-1}+p_{2 i}\right) \wp^{\prime}\left(x_{2 i-1}-x_{2 i}\right) \\
-3 \sum_{j \neq i}\left(\left(p_{2 i-1}+p_{2 j-1}\right) \wp^{\prime}\left(x_{2 i-1}-x_{2 j-1}\right)+\left(p_{2 i-1}+p_{2 j}\right) \wp^{\prime}\left(x_{2 i-1}-x_{2 j}\right)\right) .
\end{gathered}
$$


Expanding the right hand side in powers of $\varepsilon$ and comparing with (7), we obtain, in the orders $\varepsilon^{-1}, \varepsilon^{0}$ the same equations (11), (12) (this shows that the limiting procedure is consistent) and, in the next order, the equation

$$
\dot{\alpha}_{i}=-6 \alpha_{i} \sum_{j \neq i} \wp^{\prime}\left(x_{2 i-1}-x_{2 j-1}\right)-\frac{3}{2} \sum_{j \neq i}\left(\delta_{i}^{-1}-\delta_{j}^{-1}\right) \delta_{j}^{2} \wp^{\prime \prime \prime}\left(x_{2 i-1}-x_{2 j-1}\right)-12 \delta_{i}^{-3} \beta_{i, 2} .
$$

The consistency with the $t_{3}$-evolution also implies that it should hold

$$
\dot{p}_{2 i-1}+\dot{p}_{2 i}=O\left(\varepsilon^{2}\right)
$$

We have from the second equation in (8) :

$$
\begin{aligned}
-\left(\dot{p}_{2 i-1}+\dot{p}_{2 i}\right)= & 3 p_{2 i-1} \sum_{j \neq i}\left(\wp^{\prime}\left(x_{2 i-1}-x_{2 j-1}\right)+\wp^{\prime}\left(x_{2 i-1}-x_{2 j}\right)\right) \\
& +3 p_{2 i} \sum_{j \neq i}\left(\wp^{\prime}\left(x_{2 i}-x_{2 j-1}\right)+\wp^{\prime}\left(x_{2 i}-x_{2 j}\right)\right) \\
& +3 \sum_{j \neq i} p_{2 j-1} \wp^{\prime}\left(x_{2 i-1}-x_{2 j-1}\right)+3 \sum_{j \neq i} p_{2 j} \wp^{\prime}\left(x_{2 i-1}-x_{2 j}\right) \\
& +3 \sum_{j \neq i} p_{2 j-1} \wp^{\prime}\left(x_{2 i}-x_{2 j-1}\right)+3 \sum_{j \neq i} p_{2 j} \wp^{\prime}\left(x_{2 i}-x_{2 j}\right) .
\end{aligned}
$$

On the first glance, the right hand side is $O(1)$. However, an accurate $\varepsilon$-expansion shows that the terms $O(1)$ and $O(\varepsilon)$ cancel and (15) indeed holds.

It turns out that the $t_{3}$-flow can be restricted to the submanifold $\mathcal{B}_{n}=\lim _{\varepsilon \rightarrow 0} \mathcal{B}_{n}(\varepsilon)$. In this way, one can derive equations of motion for $x_{2 i-1}$, i.e. the equations connecting $\ddot{x}_{2 i-1}, \dot{x}_{2 i-1}$ and $x_{2 i-1}$. We have from (9):

$$
\ddot{x}_{2 i-1}=-6 \delta_{i}^{-1} \dot{\alpha}_{i}+6 \alpha_{i} \delta_{i}^{-2} \dot{\delta}_{i}+6 \sum_{j \neq i}\left(\dot{x}_{2 i-1}-\dot{x}_{2 j-1}\right) \wp^{\prime}\left(x_{2 i-1}-x_{2 j-1}\right) .
$$

Plugging here $\dot{\alpha}_{i}$ from (14) with $\beta_{i, 2}$ from (13), we see that all $\delta_{i}$ cancel and the following equations of motion for $x_{i}$ hold:

$$
\ddot{x}_{i}+6 \sum_{j \neq i}\left(\dot{x}_{i}+\dot{x}_{j}\right) \wp^{\prime}\left(x_{i}-x_{j}\right)-72 \sum_{j \neq i} \sum_{k \neq i} \wp\left(x_{i}-x_{j}\right) \wp^{\prime}\left(x_{i}-x_{k}\right)+6 \sum_{j \neq i} \wp^{\prime \prime \prime}\left(x_{i}-x_{j}\right)=0
$$

(here $i, j, k$ are odd numbers running from 1 to $2 n-1)$. Using the identity $\wp^{\prime \prime \prime}(x)=$ $12 \wp(x) \wp^{\prime}(x)$, we represent them in the form

$$
\ddot{x}_{i}+6 \sum_{j \neq i}\left(\dot{x}_{i}+\dot{x}_{j}\right) \wp^{\prime}\left(x_{i}-x_{j}\right)-72 \sum_{j \neq k \neq i} \wp\left(x_{i}-x_{j}\right) \wp^{\prime}\left(x_{i}-x_{k}\right)=0 .
$$

These are equations obtained in [8] (see also [10]) for dynamics of poles of elliptic solutions to the BKP equation.

In fact the calculations are considerably simplified if one puts $\delta_{i}=1$ from the very beginning, i.e. $x_{2 i}-x_{2 i-1}=\varepsilon$. This fixes the "gauge freedom" in the definition of $\mathcal{B}_{n}(\varepsilon)$. 
The coefficients $\beta_{i, a}$ become functions of $x_{2 j-1}, \alpha_{j}$ which are independent coordinates in the subspace $\mathcal{B}_{n}$. The formulas given above show that the restriction of the CalogeroMoser dynamics in $t_{3}(8)$ to $\mathcal{B}_{n}$ can be written as a first order system of equations

$$
\left\{\begin{array}{l}
\dot{x}_{2 i-1}=-6 \alpha_{i}+6 \sum_{j \neq i} \wp\left(x_{2 i-1}-x_{2 j-1}\right), \\
\dot{\alpha}_{i}=-12 \alpha_{i} \sum_{j \neq i} \wp^{\prime}\left(x_{2 i-1}-x_{2 j-1}\right)+\sum_{j \neq i} \wp^{\prime \prime \prime}\left(x_{2 i-1}-x_{2 j-1}\right) .
\end{array}\right.
$$

Excluding $\alpha_{i}$, one gets equations (18).

\section{The Lax matrix}

Let us introduce the function $\Phi(x, \lambda)$ defined as

$$
\Phi(x, \lambda)=\frac{\sigma(x+\lambda)}{\sigma(\lambda) \sigma(x)} e^{-\zeta(\lambda) x}
$$

where $\zeta(\lambda)$ is the Weierstrass $\zeta$-function, i.e. the odd function such that $\zeta^{\prime}=-\wp$. It has a simple pole at $x=0$ with residue 1 and the expansion

$$
\Phi(x, \lambda)=x^{-1}-\frac{1}{2} \wp(\lambda) x-\frac{1}{6} \wp^{\prime}(\lambda) x^{2}+O\left(x^{3}\right) \quad \text { as } x \rightarrow 0 .
$$

The function $\Phi$ has the following quasiperiodicity properties:

$$
\Phi\left(x+2 \omega_{\alpha}, \lambda\right)=e^{2\left(\zeta\left(\omega_{\alpha}\right) \lambda-\zeta(\lambda) \omega_{\alpha}\right)} \Phi(x, \lambda), \quad \alpha=1,2 .
$$

In what follows we will often suppress the second argument of $\Phi$ writing simply $\Phi(x)=$ $\Phi(x, \lambda)$. We will also need the $x$-derivatives $\Phi^{\prime}(x, \lambda)=\partial_{x} \Phi(x, \lambda), \Phi^{\prime \prime}(x, \lambda)=\partial_{x}^{2} \Phi(x, \lambda)$ and so on.

The Lax matrix of the elliptic Calogero-Moser system reads

$$
L_{j k}^{\mathrm{CM}}=L_{j k}^{\mathrm{CM}}(\lambda)=p_{j} \delta_{j k}+\left(1-\delta_{j k}\right) \Phi\left(x_{j}-x_{k}, \lambda\right) .
$$

It is an $N \times N$ matrix depnding on the spectral parameter $\lambda$. We are going to restrict it to $\mathcal{B}_{n}(\varepsilon)$ as $\varepsilon \rightarrow 0$ (with $\delta_{i}=1$ ). It is convenient to re-denote $\alpha_{i} \rightarrow \alpha_{2 i-1}$. The result is a $2 n \times 2 n$ matrix which is represented as a block matrix with $2 \times 2$ blocks numbered by odd numbers $j, k$ running from 1 to $2 n-1$ :

$$
\begin{aligned}
L_{j k}^{(\varepsilon)}=\varepsilon^{-1} & \bar{e} \delta_{j k}+\left(1-\delta_{j k}\right) e \Phi\left(x_{j}-x_{k}\right) \\
& +\varepsilon\left[\left(\alpha_{j} \sigma_{3}+\frac{i}{2} \wp(\lambda) \sigma_{2}\right) \delta_{j k}-i\left(1-\delta_{j k}\right) \sigma_{2} \Phi^{\prime}\left(x_{j}-x_{k}\right)\right]+O\left(\varepsilon^{2}\right),
\end{aligned}
$$

where the matrices $e, \bar{e}$ are

$$
e=\sigma_{0}+\sigma_{1}=\left(\begin{array}{ll}
1 & 1 \\
1 & 1
\end{array}\right), \quad \bar{e}=\sigma_{3}-i \sigma_{2}=\left(\begin{array}{cc}
1 & -1 \\
1 & -1
\end{array}\right)
$$


and $\sigma_{0}, \sigma_{1}, \sigma_{2}, \sigma_{3}$ are the standard Pauli matrices ( $\sigma_{0}$ is the unity matrix). For example $(n=2)$ :

$$
L^{(\varepsilon)}(\lambda)=\left(\begin{array}{cccc}
\varepsilon^{-1}+\alpha_{1} \varepsilon & -\varepsilon^{-1}+\frac{1}{2} \wp(\lambda) \varepsilon & \Phi\left(x_{13}\right) & \Phi\left(x_{13}\right)-\varepsilon \Phi^{\prime}\left(x_{13}\right) \\
\varepsilon^{-1}-\frac{1}{2} \wp(\lambda) \varepsilon & -\varepsilon^{-1}-\alpha_{1} \varepsilon & \Phi\left(x_{13}\right)+\varepsilon \Phi^{\prime}\left(x_{13}\right) & \Phi\left(x_{13}\right) \\
\Phi\left(x_{31}\right) & \Phi\left(x_{31}\right)-\varepsilon \Phi^{\prime}\left(x_{31}\right) & \varepsilon^{-1}+\alpha_{3} \varepsilon & -\varepsilon^{-1}+\frac{1}{2} \wp(\lambda) \varepsilon \\
\Phi\left(x_{31}\right)+\varepsilon \Phi^{\prime}\left(x_{31}\right) & \Phi\left(x_{31}\right) & \varepsilon^{-1}-\frac{1}{2} \wp(\lambda) \varepsilon & -\varepsilon^{-1}-\alpha_{3} \varepsilon \\
c & +O\left(\varepsilon^{2}\right), &
\end{array}\right.
$$

where $x_{j k}=x_{j}-x_{k}$. It can be seen that

$$
\lim _{\varepsilon \rightarrow 0} \operatorname{det}_{2 n \times 2 n}\left(L^{(\varepsilon)}(\lambda)-z I\right)=R(z, \lambda)
$$

exists and $\operatorname{det}_{2 n \times 2 n}\left(L^{(\varepsilon)}(\lambda)-z I\right)$ is $O(1)$ as $\varepsilon \rightarrow 0$. Here $I$ is the unity matrix. The equation of the spectral curve is $R(z, \lambda)=0$. Presumably, this is the same equation as

$$
\operatorname{det}_{n \times n} \mathcal{L}(z, \lambda)=0,
$$

where $\mathcal{L}(z, \lambda)$ is the $n \times n$ Lax matrix for pole dynamics of elliptic solutions to the BKP equation given by [8]

$$
\begin{aligned}
\mathcal{L}_{j k}(z, \lambda)=( & \left.-\dot{x}_{j}+6 \sum_{l \neq j} \wp\left(x_{j k}\right)-3\left(z^{2}-\wp(\lambda)\right)\right) \delta_{j k} \\
& \left.-6\left(1-\delta_{j k}\right) \Phi^{\prime}\left(x_{j k}\right)\right)-6 z\left(1-\delta_{j k}\right) \Phi\left(x_{j k}\right) .
\end{aligned}
$$

However, we were able to check this explicitly only in the case $n=1$. The equations of motion (18) are equivalent to the commutation representation for the matrix $\mathcal{L}(z, \lambda)$ in the form of the Manakov's triple [11]:

$$
\dot{\mathcal{L}}+[\mathcal{L}, \mathcal{M}]=\mathcal{P} \mathcal{L}
$$

with some matrix $\mathcal{M}=\mathcal{M}(z, \lambda)$ depending on the dynamical variables, where $\mathcal{P}$ is a traceless matrix.

\section{Concluding remarks}

We have shown that the third Hamiltonian flow of the elliptic Calogero-Moser model preserves the configuration in which the particles join in pairs, with two particles in each pair being placed in one and the same point. We have also derived the effective equations of motion for the pairs and have shown that they coincide with equations of motion for poles of elliptic solutions to the BKP equation. A problem for the future is to find out whether these equations of motion are Hamiltonian or not.

An advanced approach for analyzing collisions of Calogero-Moser particles (in the model with rational potential) was developed in [12, 13]. It essentially consists in certain completion of the phase space which allows one to describe configurations in which coordinates of some particles coincide. It would be very interesting to establish connections with the approach of that papers, at least in the rational case, where it is available. 


\section{Acknowledgments}

The author thanks I. Krichever and D. Rudneva for discussions. The work was performed at the Steklov Mathematical Institute of Russian Academy of Sciences, Moscow, and was supported by the Russian Science Foundation under grant 19-11-00062.

\section{References}

[1] F. Calogero, Solution of the one-dimensional N-body problems with quadratic and/or inversely quadratic pair potentials, J. Math. Phys. 12 (1971) 419-436.

[2] F. Calogero, Exactly solvable one-dimensional many-body systems, Lett. Nuovo Cimento 13 (1975) 411-415.

[3] J. Moser, Three integrable Hamiltonian systems connected with isospectral deformations, Adv. Math. 16 (1975) 197-220.

[4] M.A. Olshanetsky and A.M. Perelomov, Classical integrable finite-dimensional systems related to Lie algebras, Phys. Rep. 71 (1981) 313-400.

[5] I.M. Krichever, Elliptic solutions of the Kadomtsev-Petviashvili equation and integrable systems of particles, Funk. Anal. i Ego Pril. 14:4 (1980) 45-54 (in Russian); English translation: Functional Analysis and Its Applications 14:4 (1980) 282 - 290.

[6] E. Date, M. Jimbo, M. Kashiwara and T. Miwa, Transformation groups for soliton equations: Nonlinear integrable systems - classical theory and quantum theory (Kyoto, 1981). Singapore: World Scientific, 1983, 39-119.

[7] E. Date, M. Jimbo, M. Kashiwara and T. Miwa, Transformation groups for soliton equations IV. A new hierarchy of soliton equations of KP type, Physica D 4D (1982) 343-365.

[8] D. Rudneva and A. Zabrodin, Dynamics of poles of elliptic solutions to BKP equation, Journal of Physics A: Math. Theor. 53 (2020) 075202.

[9] H. Ujino, M. Wadati and K. Hikami, The quantum Calogero-Moser model: algebraic structures, J. Phys. Soc. Japan 62 (1993) 3035-3043.

[10] A. Zabrodin, Elliptic solutions to integrable nonlinear equations and many-body systems, Journal of Geometry and Physics 146 (2019) 103506.

[11] S. Manakov, Method of inverse scattering problem and two-dimensional evolution equations, Uspekhi Mat. Nauk 31 (1976) 245-246.

[12] D. Kazhdan, B. Kostant and S. Sternberg, Hamiltonian group actions and dynamical systems of Calogero type, Comm. Pure Appl. Math. 31 (1978) 481-507.

[13] G. Wilson, Collisions of Calogero-Moser particles and an adelic Grassmannian, Invent. Math. 133 (1998) 1-41. 